\title{
Ward-Takahashi Identity on the Light Front**
}

\author{
H. W. L. Naus ${ }^{a}$, J. P. B. C. de Melo ${ }^{b}$ and T. Frederico ${ }^{c}$ \\ ${ }^{a}$ Institute for Theoretical Physics, University of Hannover \\ Appelstr. 2, 30167 Hannover, Germany \\ ${ }^{b}$ Instituto de Física, Universidade de São Paulo \\ 01498-970 São Paulo, São Paulo, Brazil \\ ${ }^{c}$ Dep. de Física, Instituto Tecnológico da Aeronáutica, \\ Centro Técnico Aeroespacial, 12.228-900 São José dos Campos, São Paulo, Brazil
}

October 12,2018

\begin{abstract}
The Ward-Takahashi identity, reflecting local gauge invariance, is perturbatively verified for a boson model in light front field theory. A careful integration over the light front energy, corresponding to exactly taking into account pair terms, which are the contributions of the zero longitudinal momentum mode, is crucial to obtain this result. Furthermore, the one-loop boson form factors are calculated for arbitrary off-shell momenta.
\end{abstract}

*Dedicated to Prof. John A. Tjon on the occasion of his 60th birthday.

${ }^{*}$ Submitted in Few Body Systems 


\section{Introduction}

Ward-Takahashi (WT) identities [1], 2] are among the most interesting consequences of local $U(1)$ gauge invariance in quantum field theory. It appears therefore surprising that they have not been addressed in light front field theory. One reason may be that a formal proof [1, 2, 3, exploiting equal time canonical commutation relations, cannot be taken over to the light front. Front form dynamics can only be described as a constrained system and not be quantized canonically. In fermionic theories, for example, some components of the spinor fields are not independent but necessarily obey constraint equations. These "bad" components also enter in the electromagnetic operators, which, in turn, lead to bad currents. Recall the simplest WT identity: the relation between electromagnetic vertex and propagator. Because current and electromagnetic vertex are evidently related, the question arises whether the WT identity actually holds on the light front.

Instead of a formal and more general approach to this problem, we first study a simple model using perturbation theory. We explicitly calculate vertex function and self-energy of a charged boson; its structure arises from its interaction with a neutral and a charged boson. Our approach is based on a series of papers by Chang, Root and Yan [4, 5, 6, 7], where it was demonstrated that light front perturbative field theory amounts to using the Feynman rules and then first integrating over the light front energy, $p^{-}$, in momentum integrals. It can be compared to (re-)deriving time ordered perturbation theory by integration over the energy variable $p^{0}=E$. At this point several remarks are in order. First, special care is needed in the actual $p^{-}$integration since a too naive approach yields wrong answers [7]. We will come back to this point below. Second, this simple recipe cannot readily be applied to theories with massless particles and/or gauge theories. Since we consider massive bosons and we do not include radiative corrections this poses no restriction for our work. Finally, in recent years the modes of vanishing light front momentum $p^{+}$have attracted much attention (see, e.g. [8] and references therein). It is a priori not clear whether the recipe also works in this zero mode sector. We speculate, however, that the careful integration mentioned above, indeed renders this problem for massive virtual particles.

Recently, also the role of the pair (creating) terms has been discussed for the same boson model [9]. It was shown that the pair terms survive even in the limit of zero light front momentum $p^{+}$and that they are necessary to obtain a covariant current. Here we extend this work to general off-shell momenta, consider WT identities and confirm the relevance of the pair terms. Furthermore, all components of the electromagnetic vertex operator in an 
arbitrary Lorentz-frame are constructed. Obviously, our calculation produces the on-shell form factor, its off-shell extension, as well as the additional off-shell form factor. In refs. [10, 11] similar issues for the nucleon, however using a meson field theory in the instant form, have been investigated.

In this paper, we again employ the "dislocation of pole integration" developed in [9]. On the other hand, in contrast to the latter work we start from one basic integral which, after regularization, can be completely performed. Thus after the $p^{-}$integration, the integrations over the other components of $p$ are carried out. In this way we can immediately calculate all components of the boson current, demonstrating that for this simple model the term "bad component" is somewhat artificial. Since we are interested in WT identities gauge invariant regularization and renormalization procedures are preferred, ruling out excessively naive momentum cut-offs.

\section{Basic Techniques}

We first develop the basic calculational techniques. Consider the following integral in light front variables

$$
I_{1}\left(M^{2}\right)=\int d^{4} p \frac{1}{p^{2}-M^{2}+i \epsilon}=\frac{1}{2} \int d^{2} p_{\perp} \int d p^{+} \int d p^{-} \frac{1}{p^{+} p^{-}-p_{\perp}^{2}-M^{2}+i \epsilon},
$$

where one needs to integrate first over the light front energy $p^{-}$. Recall that a naive light front integration immediately would yield zero [7], obviously the wrong answer. Furthermore, note that this integral is divergent. In order to regularize the integral in the ultraviolet domain we introduce a regulator $M_{R}[12]$ :

$$
I_{1}^{r e g}\left(M^{2}, M_{R}^{2}\right)=\frac{1}{2} \int d^{2} p_{\perp} \int d p^{+} \int d p^{-} \frac{1}{p^{+} p^{-}-p_{\perp}^{2}-M^{2}+i \epsilon}\left(\frac{-M_{R}^{2}}{p^{+} p^{-}-p_{\perp}^{2}-M_{R}^{2}+i \epsilon}\right)^{2} .
$$

At the end of the day, the regulator is supposed to approach infinity. Let us introduce the notation $\tilde{M}^{2}=M^{2}+p_{\perp}^{2}$ and $\tilde{M}_{R}^{2}=M_{R}^{2}+p_{\perp}^{2}$. To prepare the $p^{-}$integration one separates a factor $1 / p^{+}$, e.g.

$$
\frac{1}{p^{+} p^{-}-p_{\perp}^{2}-M^{2}+i \epsilon}=\frac{1}{p^{+}} \frac{1}{p^{-}-\frac{\tilde{M}^{2}-i \epsilon}{p^{+}}} .
$$

Clearly, this procedure may cause problems for $p^{+}=0$, the zero momentum mode. In order to regulate this infrared singularity we dislocate the other poles [9]:

$$
\frac{1}{p^{+} p^{-}-p_{\perp}^{2}-M_{R}^{2}+i \epsilon} \rightarrow \frac{1}{p^{+} \pm \delta} \frac{1}{p^{-}-\frac{\tilde{M}_{R}^{2}-i \epsilon}{p^{+} \pm \delta}}
$$

with a small parameter $\delta$. Then the regularized integral reads

$$
I_{1}^{r e g}\left(M^{2}, M_{R}^{2}\right)=\frac{1}{2} \int d^{2} p_{\perp} \int d p^{+} \frac{1}{p^{+}\left(p^{+}+\delta\right)\left(p^{+}-\delta\right)} \int d p^{-} \frac{1}{p^{-}-\frac{\tilde{M}^{2}-i \epsilon}{p^{+}}} \frac{M_{R}^{2}}{p^{-}-\frac{\tilde{M}_{R}^{2}-i \epsilon}{p^{+}+\delta}} \frac{M_{R}^{2}}{p^{-}-\frac{\tilde{M}_{R}^{2}-i \epsilon}{p^{+}-\delta}} .
$$


With a modest amount of foresight we do not include $\delta$ as an additional argument of $I_{1}^{r e g}$. At this point we integrate over $p^{-}$; the result can be written as

$$
I_{1}^{r e g}\left(M^{2}, M_{R}^{2}\right)=i \pi M_{R}^{4} \int d^{2} p_{\perp} \frac{1}{\tilde{M}_{R}^{2} \delta} \int_{0}^{\delta} d p^{+} \frac{p^{+}-\delta}{p^{+}\left(M_{R}^{2}-M^{2}\right)+\tilde{M}^{2} \delta} .
$$

Doing the $p^{+}$integration yields

$$
I_{1}^{r e g}\left(M^{2}, M_{R}^{2}\right)=\frac{i \pi M_{R}^{4}}{M_{R}^{2}-M^{2}} \int d^{2} p_{\perp} \frac{1}{\tilde{M}_{R}^{2}}\left(1-\frac{\tilde{M}_{R}^{2}}{\tilde{M}_{R}^{2}-M^{2}} \ln \frac{\tilde{M}_{R}^{2}}{\tilde{M}^{2}}\right) .
$$

Note that this expression does not depend anymore on the infrared regulator $\delta$, a forteriori justifying the procedure. The "dislocation of the pole" is vanishing small, thus in fact the contribution to the integration arises from the momentum $p^{+}$near zero. This zero momentum mode can be interpreted as a pair term for finite $\delta$. Finally, we integrate over the transverse momenta and we obtain

$$
I_{1}^{r e g}\left(M^{2}, M_{R}^{2}\right)=i \pi^{2} M_{R}^{4}\left(\frac{M^{2}}{\left(M^{2}-M_{R}^{2}\right)^{2}} \ln \frac{M_{R}^{2}}{M^{2}}+\frac{1}{M^{2}-M_{R}^{2}}\right)
$$

Here the quadratic divergence explicitly shows up.

This result can indeed be considered as basic because other integrals, for example,

$$
I_{2}^{r e g}\left(M^{2}, M_{R}^{2}\right)=\frac{1}{2} \int d^{2} p_{\perp} \int d p^{+} \int d p^{-}\left(\frac{1}{p^{+} p^{-}-p_{\perp}^{2}-M^{2}+i \epsilon}\right)^{2} \frac{-M_{R}^{2}}{p^{+} p^{-}-p_{\perp}^{2}-M_{R}^{2}+i \epsilon}
$$

can readily be obtained from it. Note that we have already regularized $I_{2}$; now we immediately get

$$
I_{2}^{r e g}\left(M^{2}, M_{R}^{2}\right)=-\frac{M_{R}^{2}}{M^{4}} I_{1}^{r e g}\left(M_{R}^{2}, M^{2}\right)=i \pi^{2} M_{R}^{2}\left(\frac{M_{R}^{2}}{\left(M^{2}-M_{R}^{2}\right)^{2}} \ln \frac{M_{R}^{2}}{M^{2}}+\frac{1}{M^{2}-M_{R}^{2}}\right) .
$$

Indeed $I_{2}$ has a logarithmic divergence. Herewith, we also find the convergent integral

$$
\begin{aligned}
I_{3}\left(M^{2}\right) & =\frac{1}{2} \int d^{2} p_{\perp} \int d p^{+} \int d p^{-}\left(\frac{1}{p^{+} p^{-}-p_{\perp}^{2}-M^{2}+i \epsilon}\right)^{3} \\
& =\lim _{M_{R}^{2} \rightarrow \infty} \frac{1}{2} \frac{\partial}{\partial M^{2}} I_{2}^{r e g}\left(M^{2}, M_{R}^{2}\right)=\frac{-i \pi^{2}}{2 M^{2}}
\end{aligned}
$$

which is in agreement with the result of ref. 河.

Finally, we consider the regularized integrals

$$
I_{k}^{r e g}\left(q, M^{2}, M_{R}^{2}\right)=\frac{1}{2} \int d^{2} p_{\perp} \int d p^{+} \int d p^{-}\left(\frac{1}{p^{2}+2 p q-M^{2}+i \epsilon}\right)^{k}\left(\frac{-M_{R}^{2}}{p^{2}+2 p q-M_{R}^{2}+i \epsilon}\right)^{3-k}
$$

with $k=1,2$. Since shifts in the momenta are allowed in these convergent expressions, we easily verify

$$
I_{k}^{r e g}\left(q, M^{2}, M_{R}^{2}\right)=\left(\frac{M_{R}^{2}}{M_{R}^{2}+q^{2}}\right)^{3-k} I_{k}^{r e g}\left(M^{2}+q^{2}, M_{R}^{2}+q^{2}\right) .
$$


Similarly, we obtain the analogous expression for the convergent integral

$$
I_{3}\left(q, M^{2}\right)=\frac{1}{2} \int d^{2} p_{\perp} \int d p^{+} \int d p^{-}\left(\frac{1}{p^{2}+2 p q-M^{2}+i \epsilon}\right)^{3}=\frac{-i \pi^{2}}{2\left(M^{2}+q^{2}\right)} .
$$

We conclude that despite the first integration over $p^{-}$all the results are manifestly covariant. Again we confirm the connection between a careful $p^{-}$integration, in this case via dislocating coinciding poles, and covariance [थ]. Of course, the use of a covariant regularization procedure is also crucial in this respect.

\section{Vertex Function, Self-Energy and Ward-Takahashi Identity}

With the results obtained so far, the calculation of the vertex function and self-energy of the composite boson is standard. At this point, light front aspects do not explicitly show up anymore since they have already be taken care of in the evaluation of the relevant integrals. Thus the whole calculation now appears covariant.

The one-loop irreducible vertex function for the boson model [9] reads

$$
\Gamma_{\mu}=e\left[\left(p+p^{\prime}\right)_{\mu}+\Lambda_{\mu}\right]
$$

with the one-loop $\left(O\left(g^{2}\right)\right)$ vertex correction

$$
\Lambda_{\mu}=-g^{2} \int \frac{d^{4} k}{(2 \pi)^{4}} \frac{i^{3}\left(2 k-p-p^{\prime}\right)_{\mu}}{\left((k-p)^{2}-m^{2}+i \epsilon\right)\left(\left(k-p^{\prime}\right)^{2}-m^{2}+i \epsilon\right)\left(k^{2}-m^{2}+i \epsilon\right)} .
$$

The masses of charged and neutral constituents are taken to be equal and are denoted by $m$. We do not consider the external boson to be on its mass shell yet, thus in general $p^{2} \neq M^{2}, p^{2} \neq M^{2}$. Combining the denominators using the Feynman trick, we get

$$
\Lambda_{\mu}=2 i g^{2} \int_{0}^{1} d x \int_{0}^{x} d y \int \frac{d^{4} k}{(2 \pi)^{4}} \frac{\left(2 k-p-p^{\prime}\right)_{\mu}}{\left(k^{2}+2 k \xi-m_{0}^{2}+i \epsilon\right)^{3}}
$$

where $\xi=y\left(p^{\prime}-p\right)-x p^{\prime}$ and $m_{0}^{2}=m^{2}+\left(p^{\prime 2}-p^{2}\right) y-p^{\prime 2} x$. Below we will also use $q=p^{\prime}-p$. The momentum integral is convergent and using the results derived above we obtain

$$
\Lambda_{\mu}=\frac{i^{2} g^{2} \pi^{2}}{(2 \pi)^{4}} \int_{0}^{1} d x \int_{0}^{x} d y \frac{\left(p+p^{\prime}\right)_{\mu}+2 \xi_{\mu}}{\xi^{2}+m_{0}^{2}-i \epsilon}
$$

After rearranging we identify the form factors $F_{1}$ and $F_{2}$

$$
\Gamma_{\mu}=e\left[F_{1}\left(q^{2}, p^{2}, p^{\prime 2}\right)\left(p^{\prime}+p\right)_{\mu}+F_{2}\left(q^{2}, p^{2}, p^{2}\right)\left(p^{\prime}-p\right)_{\mu}\right]
$$


where $F_{1}\left(q^{2}, p^{2}, p^{\prime 2}\right)=1+f_{1}\left(q^{2}, p^{2}, p^{2}\right), F_{2}\left(q^{2}, p^{2}, p^{\prime 2}\right)=f_{2}\left(q^{2}, p^{2}, p^{\prime 2}\right)$ and

$$
\begin{aligned}
& f_{1}=\frac{g^{2} \pi^{2}}{(2 \pi)^{4}} \int_{0}^{1} d x \int_{0}^{x} d y \frac{x-1}{\left(p^{\prime 2}-p^{2}\right) y-p^{\prime 2} x+y^{2} q^{2}+\left(p^{2}-q^{2}-p^{\prime 2}\right) x y+x^{2} p^{\prime 2}+m^{2}-i \epsilon} \\
& f_{2}=\frac{g^{2} \pi^{2}}{(2 \pi)^{4}} \int_{0}^{1} d x \int_{0}^{x} d y \frac{x-2 y}{\left(p^{\prime 2}-p^{2}\right) y-p^{\prime 2} x+y^{2} q^{2}+\left(p^{2}-q^{2}-p^{\prime 2}\right) x y+x^{2} p^{\prime 2}+m^{2}-i \epsilon}
\end{aligned}
$$

The one-loop $\left(O\left(g^{2}\right)\right)$ self-energy is given by

$$
\begin{aligned}
-i \Sigma\left(p^{2}\right) & =g^{2} \int \frac{d^{4} k}{(2 \pi)^{4}} \frac{i}{\left(k+\frac{p}{2}\right)^{2}-m^{2}+i \epsilon} \frac{i}{\left(k-\frac{p}{2}\right)^{2}-m^{2}+i \epsilon} \\
& =-g^{2} \int_{0}^{1} d x \int \frac{d^{4} k}{(2 \pi)^{4}} \frac{1}{\left(k^{2}+2 k \eta-\mu^{2}+i \epsilon\right)^{2}},
\end{aligned}
$$

where $\eta=\left(x-\frac{1}{2}\right) p$ and $\mu^{2}=m^{2}-\frac{1}{4} p^{2}$. This momentum integral is divergent and we regularize as described above and get

$$
-i \Sigma\left(p^{2}\right)=\frac{-g^{2}}{(2 \pi)^{4}} \int_{0}^{1} d x I_{2}^{r e g}\left(\eta, \mu^{2}, M_{R}^{2}\right)=\frac{-g^{2}}{(2 \pi)^{4}} \int_{0}^{1} d x \frac{M_{R}^{2}}{M_{R}^{2}+\eta^{2}} I_{2}^{r e g}\left(\mu^{2}+\eta^{2}, M_{R}^{2}+\eta^{2}\right) .
$$

For large regulator $M_{R}^{2}$ we obtain

$$
\Sigma\left(p^{2}\right)=\frac{\pi^{2} g^{2}}{(2 \pi)^{4}} \int_{0}^{1} d x\left[\ln \left(\frac{M_{R}^{2}}{\mu^{2}+\eta^{2}}\right)-1\right]+\chi
$$

where $\chi$ denote terms vanishing in the limit $M_{R} \rightarrow \infty$.

At this point, i.e., before renormalization, we may already verify the WT identity

$$
\left(p^{\prime}-p\right)^{\mu} \Gamma_{\mu}=e\left[\Delta^{-1}\left(p^{2}\right)-\Delta^{-1}\left(p^{2}\right)\right],
$$

with the full propagator $\Delta$. In terms of self-energy and vertex correction it reads

$$
\left(p^{\prime}-p\right)^{\mu} \Lambda_{\mu}=\Sigma\left(p^{2}\right)-\Sigma\left(p^{2}\right) .
$$

For the lhs we find

$$
\begin{aligned}
\left(p^{\prime}-p\right)^{\mu} \Lambda_{\mu} & =-\frac{g^{2} \pi^{2}}{(2 \pi)^{4}} \int_{0}^{1} d x \int_{0}^{x} d y \frac{(1-x)\left(p^{2}-p^{2}\right)+(2 y-x) q^{2}}{\xi^{2}+m_{0}^{2}-i \epsilon} \\
& =\frac{g^{2} \pi^{2}}{(2 \pi)^{4}} \int_{0}^{1} d x \ln \left(\frac{m^{2}-p^{\prime 2} x+x^{2} p^{\prime 2}-i \epsilon}{m^{2}-p^{2} x+x^{2} p^{2}-i \epsilon}\right) .
\end{aligned}
$$

In the rhs the infinities indeed cancel, which allows us to take the limit $M_{R} \rightarrow \infty$. Then we obtain

$$
\Sigma\left(p^{2}\right)-\Sigma\left(p^{\prime 2}\right)=\frac{g^{2} \pi^{2}}{(2 \pi)^{4}} \int_{0}^{1} d x \ln \left(\frac{m^{2}-p^{\prime 2} x+x^{2} p^{\prime 2}-i \epsilon}{m^{2}-p^{2} x+x^{2} p^{2}-i \epsilon}\right) .
$$

Thus the WT identity holds. 


\section{Renormalization and Form Factors}

In order to extract observables, e.g. on-shell form factors, perturbative renormalization is nevertheless necessary. Choosing the on-shell subtraction scheme [3, 10, 11] amounts to replacing the divergent self-energy:

$$
\Sigma\left(p^{2}\right) \rightarrow \Sigma_{R}\left(p^{2}\right)=\lim _{M_{R} \rightarrow \infty}\left[\Sigma\left(p^{2}\right)-\Sigma\left(M^{2}\right)-\left(p^{2}-M^{2}\right) \Sigma^{\prime}\left(M^{2}\right)\right]
$$

Since $\Sigma_{R}\left(M^{2}\right)=0$ and $\Sigma_{R}^{\prime}\left(M^{2}\right)=0$, the renormalized propagator has a pole at the physical mass, $p^{2}=M^{2}$, with residue 1. These subtractions correspond to an infinite mass renormalization and a finite wave function renormalization. The latter also induces a finite renormalization of the vertex function

$$
\Gamma_{\mu} \rightarrow \Gamma_{\mu}^{R}=e\left[F_{1}^{R}\left(q^{2}, p^{2}, p^{2}\right)\left(p+p^{\prime}\right)_{\mu}+F_{2}^{R}\left(q^{2}, p^{2}, p^{2}\right)\left(p-p^{\prime}\right)_{\mu}\right]=\Gamma_{\mu}+\left(p+p^{\prime}\right)_{\mu} \Sigma^{\prime}\left(M^{2}\right)
$$

which implies

$$
F_{1}^{R}=F_{1}-\frac{\pi^{2} g^{2}}{(2 \pi)^{4}} \int_{0}^{1} d x \frac{x(x-1)}{M^{2} x(x-1)+m^{2}}
$$

The second form factor does not change, $F_{2}^{R}=F_{2}$. One easily checks that the WT identity is also valid for the renormalized quantities. The reducible vertex function is related to the irreducible one via

$$
\left.\Delta_{0}\left(p^{2}\right) \Gamma_{\mu}^{r e d} \Delta_{0}\left(p^{2}\right)=\Delta\left(p^{2}\right) \Gamma_{\mu}^{R} \Delta_{\left(p^{2}\right.}\right),
$$

with the free propagator $\Delta_{0}\left(p^{2}\right)=\left(p^{2}-M^{2}\right)^{-1}$. It also can be written in terms of two form factors

$$
\Gamma_{\mu}^{r e d}=e\left[g_{1}\left(q^{2}, p^{2}, p^{\prime 2}\right)\left(p+p^{\prime}\right)_{\mu}+g_{2}\left(q^{2}, p^{2}, p^{\prime 2}\right)\left(p-p^{\prime}\right)_{\mu}\right]
$$

Perturbatively it follows that

$$
g_{1}\left(q^{2}, p^{2}, p^{2}\right)=F_{1}^{R}\left(q^{2}, p^{2}, p^{\prime 2}\right)+\Sigma_{R}\left(p^{2}\right) \Delta_{0}\left(p^{2}\right)+\Sigma_{R}\left(p^{2}\right) \Delta_{0}\left(p^{2}\right),
$$

and $g_{2}=F_{2}^{R}$. In the on-shell limit the additional terms vanish: $\lim _{p^{2} \rightarrow M^{2}} \Sigma_{R}\left(p^{2}\right) \Delta_{0}\left(p^{2}\right)=0$. Particularly interesting is the half off-shell case at the photon point. Then the WT identity implies $g_{1}\left(0, p^{2}, M^{2}\right)=1$, which means that even in the half off-shell case the interpretation of $g_{1}$ as charge form factor makes sense. Using the expressions given above, it is straightforward to analytically check this relation for our model.

Let us now consider the form factors of the reducible renormalized vertex function. We restrict ourselves to the half off-shell case, $p^{\prime 2}=M^{2}$, and take $q^{2}<0$ and $p^{2}<4 m^{2}$. In this way, no poles in the integrand are present 
and, consequently, no imaginary parts appear due to real two-boson production. The integrals over the Feynman parameters are evaluated numerically. We define our units via $M^{2}=1$; then we take $m^{2}=1.1, g^{2}=0.1$. In Figs. (1) and (2) we respectively present $g_{1}$ and $g_{2}$ as a function of $-q^{2}$ for different off-shell values of $p^{2}$. It is established that $g_{1}\left(0, p^{2}, M^{2}\right)=1$ and $g_{2}\left(q^{2}, M^{2}, M^{2}\right)=0$-only one form factor is present in the on-shell limit. Both form factors reflect appreciable off-shell variations. Thus also perturbative light front field theory generates these effects.

\section{Conclusion and Outlook}

We have studied a composite charged boson in the context of a simple light front field theory. The electromagnetic vertex and self-energy of the boson have been perturbatively calculated for arbitrary off-shell momenta. Up to second order in the coupling, corresponding to the one-loop approximation, we have explicitly verified the WT identity on the light front. The accurate treatment of the zero momentum mode is crucial for this result.

In the nearby future we plan to address similar light front problems for the pion, with fermionic constituents, and for a spin $1 / 2$ particle like the nucleon. In case these studies also indicate the validity of the WT identity, it would be interesting trying to establish general, nonperturbative WT identities in light front field theory.

\section{Acknowledgements}

This work was supported in part by Fundação Coordenação de Aperfeiçoamento de Pessoal de Nível Superior and the Deutscher Akademischer Austauschdienst (Probral/CAPES/DAAD project 015/95). It was also supported by the Brazilian agencies CNPq and FAPESP. H. W. L. N. acknowledges the hospitality of the Instituto Tecnológico da Aeronáutica, Centro Técnico Aeroespacial in São José dos Campos. J. P. B. C. M. acknowledges the hospitality of the Institute for Theoretical Physics, University of Hannover. The authors thank A. C. Kalloniatis and P. U. Sauer for useful discussions and a critical reading of the manuscript. 


\section{References}

[1] Ward, J. C.: Phys. Rev. 78, 182 (1950)

[2] Takahashi, Y.: Nuovo Cimento 6, 371 (1957)

[3] Itzykson, C., Zuber, J.-B.: Quantum Field Theory. New York: MacGraw-Hill 1980

[4] Chang, S.-J., Root, R. C.: Phys. Rev. D7, 1133 (1973)

[5] Chang, S.-J, Yan, T.-M.: Phys. Rev. D7, 1147 (1973)

[6] Yan, T.-M.: Phys. Rev. D7, 1760 (1973)

[7] Yan, T.-M.: Phys. Rev. D7, 1780 (1973)

[8] Przeszowski, J., Naus, H. W. L., Kalloniatis, A. C.: Phys. Rev. D54, 5135 (1996)

[9] de Melo, J. P. B. C., Sales, J. H. O., Frederico, T., Sauer, P. U., To appear in Proceedings of "XVth International Conference on Few Body Problems in Physics”, Groningen, The Netherland, 1997.

[10] Naus, H. W. L., Koch, J. H.: Phys. Rev. C36, 2459 (1987)

[11] Tiemeijer, P. C., Tjon, J. A.: Phys. Rev. C42, 599 (1990)

[12] Pauli, W., Villars, F.: Rev. Mod. Phys. 21, 434 (1949) 


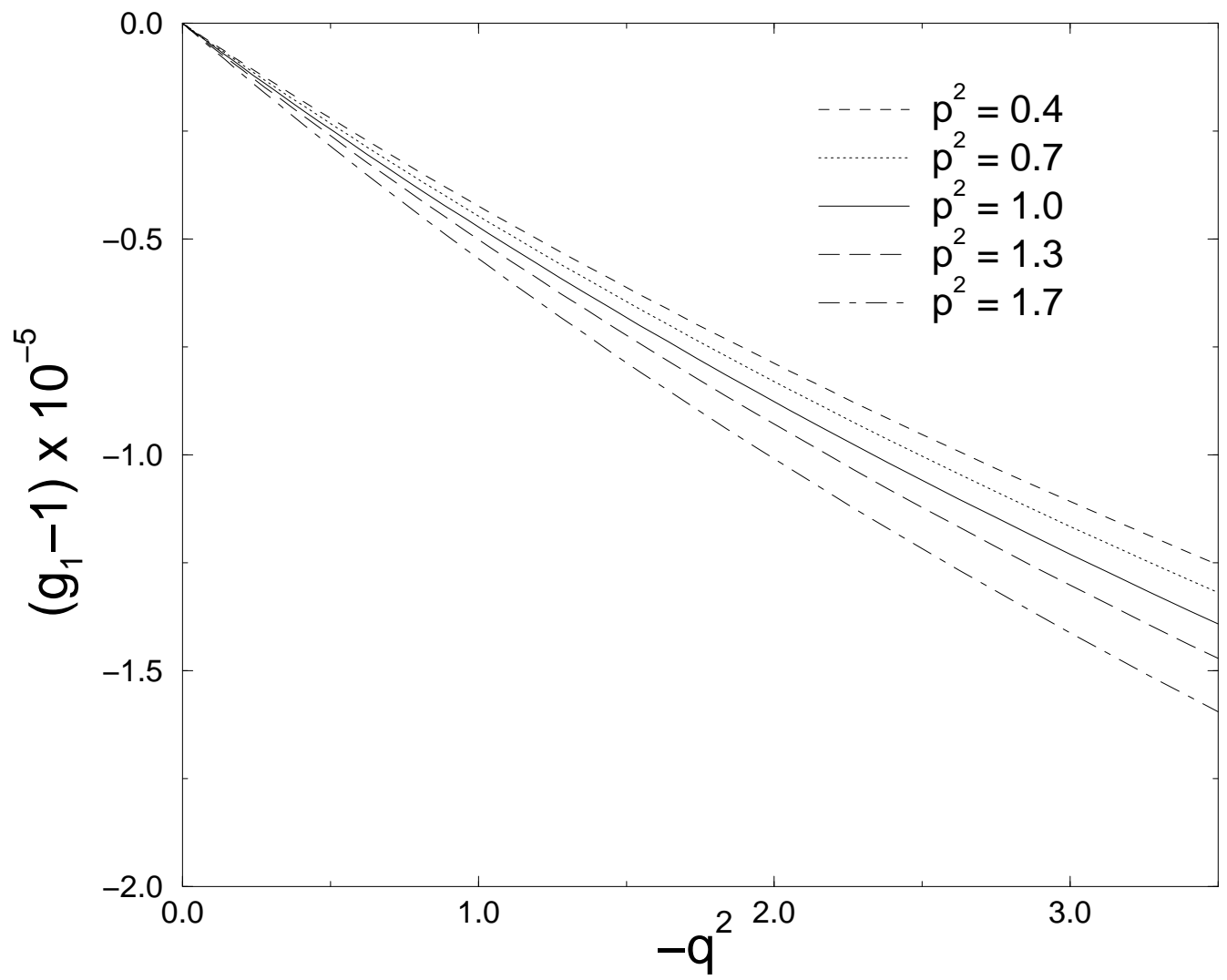

Figure 1: the form factor $g_{1}$. Note that we have plotted $\left(g_{1}-1\right)$. 


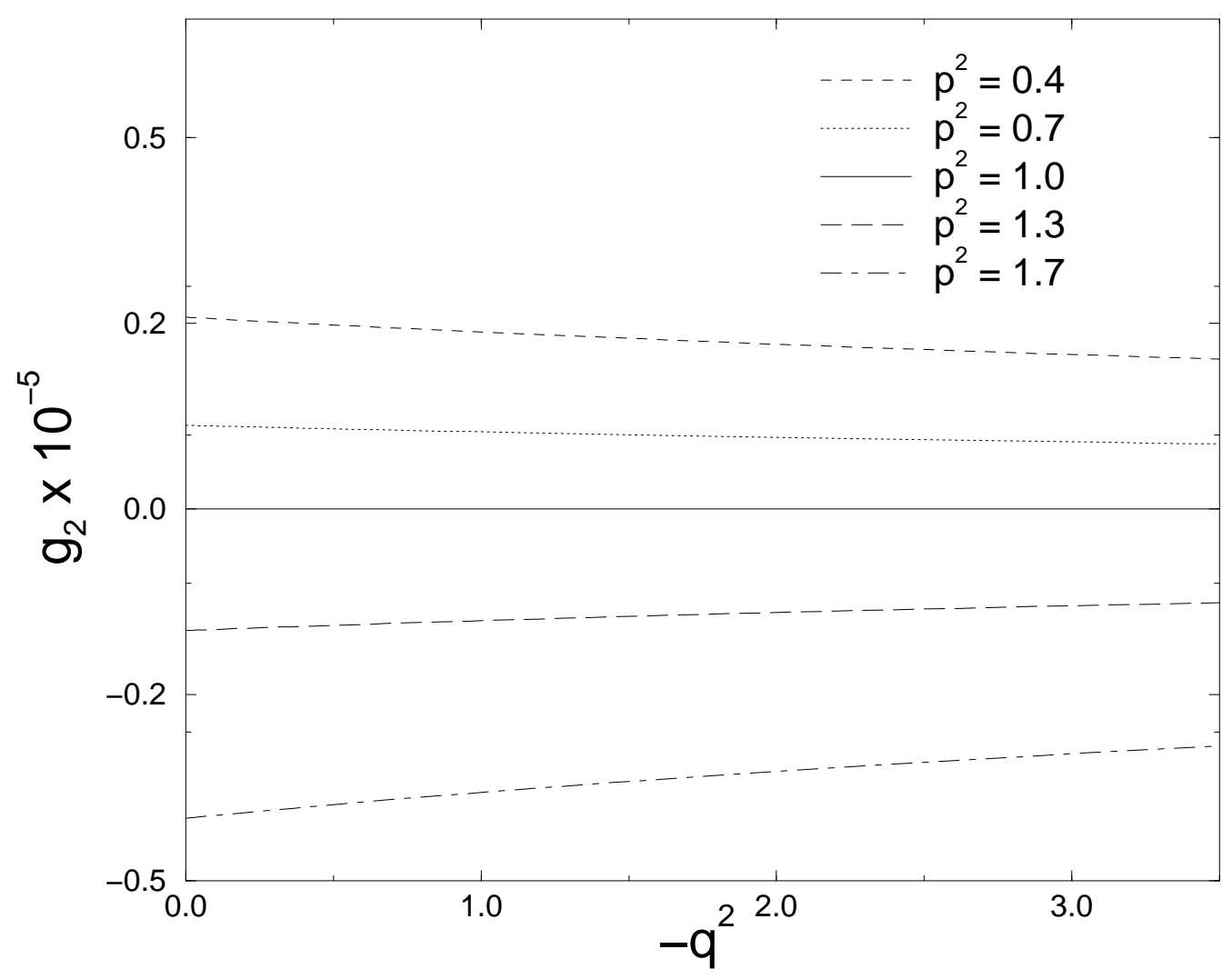

Figure 2: the form factor $g_{2}$. 